\title{
Rare case of postoperative mediastinitis following thyroidectomy
}

\author{
In Soo Cho, Moo Hyun Lee, Jihyoung Cho \\ Department of Surgery, Keimyung University School of Medicine, Daegu, Korea
}

Descending necrotizing mediastinitis (DNM) is a life-threatening complication secondary to oropharyngeal abscesses, cervical esophageal perforation, or neck infections spreading along the fascial planes into the mediastinum. Post-thyroidectomy surgical site infection is a highly unusual complication that is typically localized around the incision, but may be propagated into the mediastinum, causing DNM. We encountered a case of this rare complication after thyroidectomy. The patient was a 33-year-old woman who was admitted to our center for left hemithyroidectomy. The surgery was performed without any intraoperative events. However, she later developed fever, hypotension, and tachycardia. Imaging revealed deep neck emphysema and focal pneumomediastinum with infiltration in the anterior neck and mediastinum with abscess formation. Additional imaging at 7 days postoperatively revealed reduced fluid collection deep in the neck with minimal changes in fluid collection in the mediastinum. Esophageal perforation was excluded via an esophagogram, which lead to the conclusion that the infection may have been caused by open thyroidectomy. The patient was treated with prompt medical and surgical intervention. After treatment, she was discharged from the hospital with no further complications. We present our case report as well as a literature review of the diagnosis and treatment of this disease.

Keywords: Mediastinitis, Thyroidectomy, Postoperative complication

\section{INTRODUCTION}

Conventional open thyroidectomy is a common procedure performed worldwide that results in few life-threatening complications [1]. The lack of serious complications is most likely related to the fact that thyroid surgery is a clean surgery that does not require prophylactic antibiotics. Furthermore, the reported wound infection rate following thyroidectomy is less than $1 \%[2,3]$. Nonetheless, we encountered rare complications of descending necrotizing mediastinitis (DNM) following open thyroid lobectomy. DNM is an uncommon disease that arises as a complication of a throat in-

Received: Nov 13, 2020 Revised: Apr 1, 2021 Accepted: May 25, 2021

Correspondence to: Jihyoung Cho

Department of Surgery, Keimyung University School of Medicine,

1035 Dalgubeol-daero, Dalseo-gu, Daegu 42601, Korea

Tel: +82-53-258-4708, Fax: +82-53-258-4710

E-mail: chojh0404@dsmc.or.kr

ORCID: In Soo Cho (https://orcid.org/0000-0002-0086-3493), Moo Hyun Lee (https://orcid.org/0000-0003-3634-5230), Jihyoung Cho (https://orcid.org/00000002-1680-2919)

Copyright (C) 2021 Korean Society of Surgical Oncology

This is an Open Access article distributed under the terms of the Creative Commons Attribution Non-Commercial License (http://creativecommons.org/licenses/by-nc/4.0) which permits unrestricted non-commercial use, distribution, and reproduction in any medium, provided the original work is properly cited. fection. The disease can affect the entire mediastinum and cause severe morbidity and mortality in some cases. We herein present a rare case of post-thyroidectomy DNM. This study received approval from the Institutional Review Board of Keimyung University Dongsan Hospital (IRB No. DSMC 2020-03-080). The informed consent was waived.

\section{CASE REPORT}

A 33-year-old woman was admitted to our center for left hemithyroidectomy. She was previously diagnosed with papillary thyroid carcinoma by fine-needle aspiration. Additionally, the patient was previously diagnosed with epilepsy and was taking medication. She had a surgical history of laparoscopic distal gastrectomy due to stomach cancer 2 years prior. Although the preoperative laboratory findings were unremarkable, the preoperative ultrasonogram revealed a 7-mm sized hypoechoic nodule with microcalcifications in the left isthmus and medial aspect of the left thyroid lobe. Thus, a left hemithyroidectomy and left central compartment neck node dissection were performed. As routine antibiotic prophylaxis and drain placement are not performed at our center, our patient received neither of these treatments. No breaks in sterile technique were reported. The surgery was performed without any intraoper- 
ative events and the patient was in an acceptable condition following surgery.

The patient developed fever the first night postoperatively but experienced no specific symptoms other than pain at the surgical site; thus, she was treated with intravenous antipyretics that day. On postoperative day 1 , the patient experienced nausea and tachycardia. At 19:30, she had a high fever of over $38^{\circ} \mathrm{C}$, hypotension, and tachycardia. We suspected suspecting septic shock and admitted the patient to the surgical intensive care unit (ICU) for close monitoring and resuscitation. Empirical antibiotics, including carbapenem and vancomycin, were administered in addition to performing massive hydration using vasopressors to stabilize the patient's vital signs.

To determine the focus of the infection, neck, chest, and abdomen computed tomography (CT) scans were performed. The chest and neck CT scans revealed deep neck emphysema and focal
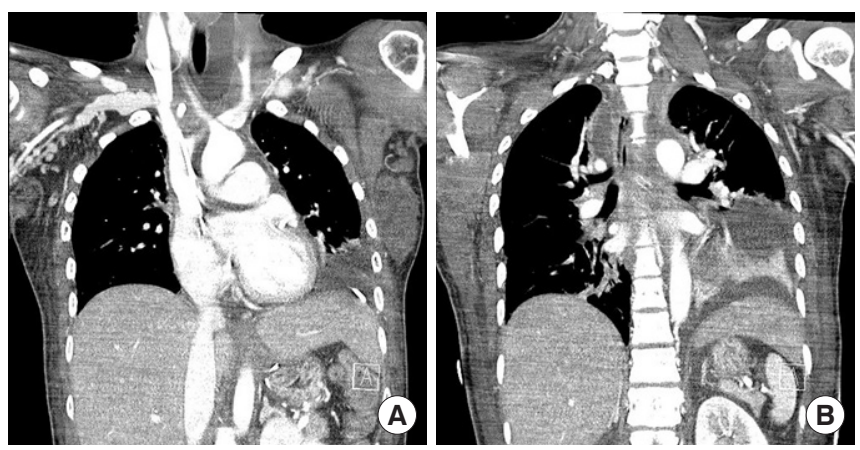

Fig. 1. (A, B) Computed tomography scan demonstrated air and fluid collection on neck and mediastinum. There was pleural effusion in left lower lobe along with atelectasis.
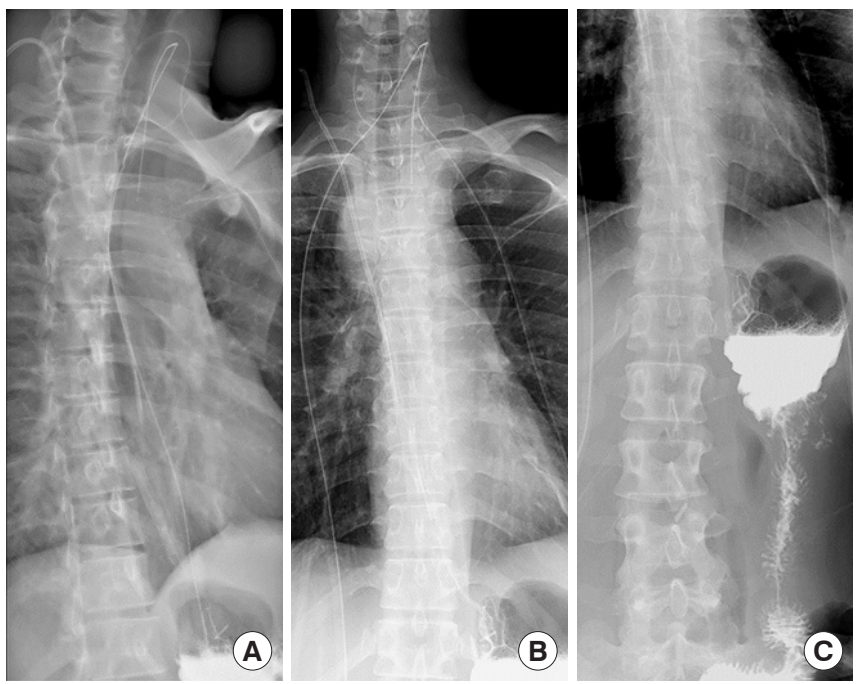

Fig. 2. (A-C) Esophagography demonstrated no contrast leakage of the esophagus to free space. pneumomediastinum with infiltration in the anterior neck and mediastinum with abscess formation (Fig. 1). We explored the infected site through previous thyroidectomy incision. Edematous changes were observed in the entire surgical field; however, there were no signs of perforation in either the esophagus or the trachea. Massive irrigation was performed and two closed-suction drains were inserted into the wound. To attain additional drainage, bedside thoracostomy was performed using the ultrasonogram. Blood and wound cultures showed no bacterial growth.

On postoperative day 7 , the patient's general condition and vital signs stabilized; hence, she was transferred to the general ward. As she was transferred to the general ward, the CT scan that was performed revealed an interval reduction in fluid collection deep in the neck with minimal changes in fluid collection in the mediastinum. A multidisciplinary approach was applied together with the thoracic surgery department: we planned a video-assisted thoracic surgery (VATS) for drainage of the fluid collected in the mediastinum.

To exclude the possibility of esophageal perforation, an esophagogram was performed using gastrografin contrast media; nonetheless, there was no leakage of the contrast, which led to the conclusion that the deep neck infection may have been caused by open thyroidectomy (Fig. 2). On the day of VATS, the patient exhibited signs of bronchospasm while general anesthesia was being induced and was retransferred to the surgical ICU for monitoring. She was extubated the following day and transferred to the general ward. She was administered empirical antibiotics and was discharged 3 weeks later without any complications. Her infection status at the 1-year follow-up was acceptable and her follow-up CT scans (Fig. 3) showed no sign of fluid collection.

\section{DISCUSSION}

Thyroidectomy is a clean procedure with a very low rate of postop-
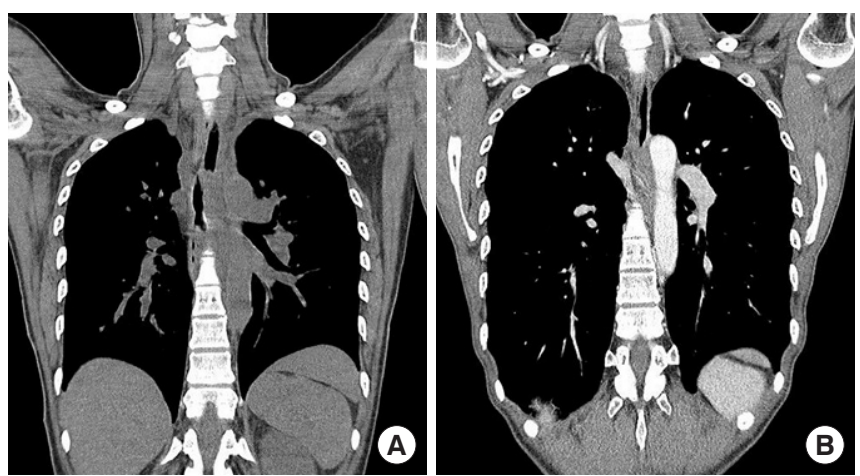

Fig. 3. (A, B) One year follow-up computed tomography scan demonstrated no evidence of inflammation of the neck and mediastinum. 
erative infection. DNM is a rare complication resulting from thyroidectomy and has thus far only been described in a few other studies; a total of 14 cases are described in the literature found through the PubMed database. One study reported six cases of fatal infection after thyroidectomy and another two studies described seven cases and one case of group A Streptococcus infection, respectively [4-6]. In our case, the abscess culture was performed 2 days after starting wide-spectrum antibiotics and cultures of the abscess cavity and blood showed no bacterial growth. Hence, it is difficult to determine the exact cause of infection.

DNM was first described by Pearse in 1938 [7]. Although rare, the disease is life-threatening and has a high mortality rate of $50 \%$. Infection of the head and neck spreads downward into the mediastinum along the cervical fascia, which is facilitated by gravity, breathing, and negative intrathoracic pressure. The deep cervical fascia is anatomically divided into three components: the pretracheal, perivascular, and prevertebral spaces. In the study by Pearse [7], the infection spread through the prevertebral space in $70 \%$ of the cases, the perivascular space in $12 \%$ of the cases, and the pretracheal space in $8 \%$ of the cases.

DNM should be diagnosed promptly and be mainly based on the clinical presentation. DNM patients typically present with severe pain in the anterior chest and neck that is sometimes accompanied by severe sepsis, or septic shock. The condition exacerbates within 24-48 hours during which prompt intervention is needed. Further, a CT scan is required to assess the extent of the disease; nonetheless, treatment should not be delayed. The CT findings in a DNM patient generally include soft tissue infiltration with loss of normal fat planes or the collection of pleural or pericardial fluid, with or without gas bubble formation, and lymphadenopathy [8]. A follow-up CT should be performed postoperatively or consecutively to assess disease progression and select an appropriate treatment.

The treatment should be performed promptly with medication or aggressive intervention. As the treatment for DNM varies, the optimal treatment has not been clearly defined. Nevertheless, the use of broad-spectrum intravenous antibiotics is essential as the disease could progress to sepsis. Additionally, the need for aggressive intervention is assessed based on the CT findings. Hasegawa et al. [9] classified DNM into three types, depending on its extent and the suggested treatment for each group. In type I, infection is localized to the upper mediastinum above the tracheal bifurcation and aggressive surgical drainage is generally not required. Type II extends to the lower anterior mediastinum, while type III reaches the anterior and lower posterior mediastinum and requires complete surgical drainage. In our case, the infection was localized to the upper mediastinum above the tracheal bifurcation so was clas- sified as type I, and the infection was improved with only transcervical drainage without mediastinal drainage.

The surgical approach for DNM varies and may include transcervical, posterolateral thoracotomy, median sternotomy, bilateral trans-sternal (clamshell) thoracotomy, or video-assisted thoracoscopy. The most commonly used approach is the combination of cervicectomy and thoracotomy [10-12]. One systematic review conducted by Prado-Calleros et al. [12] analyzed DNM case series reported in PubMed between 2009 and 2014. The review reports that the combination of cervicectomy and thoracotomy resulted in mortality ranging from $9 \%$ to $19 \%$ compared to a mortality of $47 \%$ to $50 \%$ with cervical drainage only, in cases of infection to the inferior mediastinum. Our center performed cervicectomy with thoracostomy drainage; elective VATS drainage was planned but was cancelled due to the patient's poor response to general anesthesia. Notwithstanding that thoracotomy drainage was not performed, the patient fared well without further spread of the infection.

In conclusion, post-thyroidectomy DNM is a rare but dangerous complication. Physicians should be conscious of cases with a cervical abscess and descending mediastinitis secondary to thyroidectomy. Based on previous reports and the results reported herein, the condition should be treated aggressively with appropriate cervical and mediastinal drainage in combination with optimal medical care.

\section{CONFLICT OF INTEREST}

No potential conflict of interest relevant to this article was reported.

\section{ACKNOWLEDGMENTS}

This work was supported by a National Research Foundation of Korea (NRF) grant funded by the Korea Government (MSIP) (grant No. 2018R1C1B5086617).

\section{REFERENCES}

1. Bae DS, Koo DH. A propensity score-matched comparison study of surgical outcomes in patients with differentiated thyroid cancer after robotic versus open total thyroidectomy. World J Surg 2019; 43:540-51.

2. Rosato L, Avenia N, Bernante P, De Palma M, Gulino G, Nasi PG, et al. Complications of thyroid surgery: analysis of a multicentric study on 14,934 patients operated on in Italy over 5 years. World J Surg 2004;28:271-6.

3. Spanknebel K, Chabot JA, DiGiorgi M, Cheung K, Lee S, Allen- 
dorf J, et al. Thyroidectomy using local anesthesia: a report of 1,025 cases over 16 years. J Am Coll Surg 2005;201:375-85.

4. Hardy RG, Forsythe JL. Uncovering a rare but critical complication following thyroid surgery: an audit across the UK and Ireland. Thyroid 2007;17:63-5.

5. Faibis F, Sapir D, Luis D, Laigneau P, Lepoutre A, Pospisil F, et al. Severe group a Streptococcus infection after thyroidectomy: report of three cases and review. Surg Infect (Larchmt) 2008;9:529-31.

6. Collin Y, Sirois M, Carignan A, Lawton Wackett JC. Group A Streptococcus causing descending necrotizing mediastinitis: report of a case and literature review. Surg Infect (Larchmt) 2012;13:57-9.

7. Pearse HE. Mediastinitis following cervical suppuration. Ann Surg 1938;108:588-611.

8. Scaglione M, Pinto A, Romano S, Giovine S, Sparano A, Romano L. Determining optimum management of descending necrotizing mediastinitis with CT; experience with 32 cases. Emerg Radiol 2005;11:275-80.

9. Hasegawa T, Endo S, Sohara Y. Classification of descending necrotizing mediastinitis. Ann Thorac Surg 2000;69:1296.

10. Marty-Ane CH, Alauzen M, Alric P, Serres-Cousine O, Mary H. Descending necrotizing mediastinitis. Advantage of mediastinal drainage with thoracotomy. J Thorac Cardiovasc Surg 1994;107: 55-61.

11. Corsten MJ, Shamji FM, Odell PF, Frederico JA, Laframboise GG, Reid KR, et al. Optimal treatment of descending necrotizing mediastinitis. Thorax 1997;52:702-8.

12. Prado-Calleros HM, Jimenez-Fuentes E, Jimenez-Escobar I. Descending necrotizing mediastinitis: systematic review on its treatment in the last 6 years, 75 years after its description. Head Neck 2016;38 Suppl 1:E2275-83. 